\title{
AVALIAÇÃO DO PERFIL DO SERVIÇO DE ATENÇÃO DOMICILIAR NO ESTADO DE PERNAMBUCO: CORTE 2011 $-2016$
}

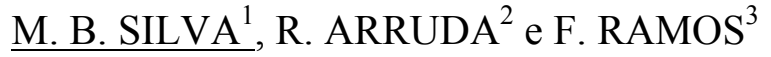 \\ ${ }^{1}$ Universidade Federal de Pernambuco, Centro de Ciências Sociais Aplicadas, Mestranda do \\ curso de Gestão e Economia da Saúde; \\ ${ }^{2}$ Universidade Federal de Pernambuco, Centro de Ciências Sociais Aplicadas, Departamento \\ de Economia; \\ ${ }^{3}$ Universidade Federal de Pernambuco, Centro de Ciências Sociais Aplicadas, Departamento \\ de Economia.
}

E-mail para contato: michelybezerra@yahoo.com.br

RESUMO - A Politica de Atenção Domiciliar, representada pelo Programa Melhor em Casa, representa uma iniciativa do Governo Federal para expandir e qualificar a Atenção Domiciliar no Sistema Único de Saúde, frente a esse cenário de transição epidemiológica e demográfica, e à crise do modelo de atenção hegemônico. Este estudo objetiva caracterizar a o perfil do Serviço de Atenção Domiciliar no estado de Pernambuco entre os anos de 2011 a 2016 no tocante ao números de internações no SUS em relação ao numero de leitos e quanto percentualmente representa o incremento desse programa ao longo desse corte histórico. Foi utilizado como método para traduzir o perfil do serviço de atenção domiciliar em Pernambuco, um estudo descritivo e exploratório onde se realizou uma análise de frequências simples das variáveis contidas no instrumento de monitoramento do "Programa Melhor em Casa" do Ministério da Saúde. A análise dos dados foi realizada por meio de estatística descritiva, dos dados retirados do programa Tab para Windows - TabWin; além do software Excel 2017. Os resultados mostram que o Nordeste possui 32,97\% dos serviços de atenção domiciliar implantada e desses $15,46 \%$ do total de SAD implantadas no nordeste estão no estado de estado de Pernambuco. 47(quarenta e sete) equipes compõem o Serviço de Atenção Domiciliar no estado, sendo a microrregião do Recife (Abreu e Lima, Jaboatão dos Guararapes e Recife) responsável por 48,94\% dessas equipes. O que se conclui com o estudo é que o serviço de atenção domiciliar tem de fato uma capacidade de descongestionar os hospitais e proporcionar a construção de nova lógica de atenção, racionalizar a utilização dos leitos hospitalares.

Palavras-chave: Assistência Domiciliar. Avaliação de Políticas Públicas. Saúde Pública. Avaliação serviço de saúde.

ABSTRACT - The Home Care Policy, represented by the Best Home Program, represents an initiative of the Federal Government to expand and qualify Home Care in the Unified Health System, in face of this scenario of epidemiological and demographic transition, and the crisis of the model of hegemonic attention. This study aims to characterize the profile of the Home Care Service in the state of Pernambuco between the years 2011 to 
2016 regarding the number of hospitalizations in the SUS in relation to the number of beds and how much represents the increment of this program along this historical cut. A descriptive and exploratory study was used as a method to translate the profile of the home care service in Pernambuco, where a simple frequency analysis of the variables contained in the monitoring tool of the "Best in-home Program" of the Ministry of Health was performed. of the data was made by means of descriptive statistics, of the data taken from the Tab program for Windows - TabWin; in addition to the software Excel 2017. The results show that the Northeast has $32.97 \%$ of the home care services implanted and of these $15.46 \%$ of the total SADs implanted in the northeast are in the state of Pernambuco. 47 (forty-seven) teams make up the Home Care Service in the state, with the micro-region of Recife (Abreu and Lima, Jaboatão dos Guararapes and Recife) accounting for $48.94 \%$ of these teams. What concludes with the study is that the home care service has in fact an ability to decongest the hospitals and provide the construction of new logic of care, rationalize the use of hospital beds.

Keywords: Home Care. Evaluation of Public Policies. Public health. Health service evaluation.

\section{INTRODUÇÃO}

A Atenção Domiciliar (AD) vem confirmando ser um novo horizonte para o Sistema Nacional de Saúde (SNS), em especial nas últimas décadas, por ser uma das alternativas para transição epidemiológica e demográfica, e à crise do modelo de atenção hegemônico de atenção a saúde hoje praticado (GALVÃO, 2011). No Brasil, essa modalidade AD vem sendo experimentada, com diversos arranjos, desde a década de 1960 (BRASIL, 2012 apud OLIVEIRA, 2016). Atualmente a AD é regido pela Portaria MS n ${ }^{\circ} 825$ de 25 de abril de 2016 (BRASIL, 2011; BRASIL, 2016).

Hoje, o SAD é visto como uma alternativa concreta de racionalizar a utilização dos leitos hospitalares, devido a problemas relativos à ineficiência, ineficácia, altos custos do sistema hospitalar e um aumento crescente da demanda em cuidados de saúde, tentativa de superar a crise do modelo de atenção hospitalar (SILVA et al., 2010; BRASIL, 2012, SILVA, 2014; ALENCAR, 2014).

Este artigo, dentro de uma perspectiva de que o SAD potencialize a utilização de leitos hospitalares, diminuindo a superlotação dos serviços de urgência/emergência do estado de Pernambuco, objetiva caracterizar o perfil do Serviço de Atenção Domiciliar no estado de Pernambuco entre os anos de 2011 a 2016 no tocante ao números de internações no SUS em relação ao numero de leitos e quanto percentualmente representa o incremento desse programa ao longo desse corte histórico.

\section{MATERIAIS E MÉTODOS}

A avaliação do SAD no estado de Pernambuco, foi feita através de pesquisa bibliográfica e quantitativa (estatística descritiva), tendo como embasamento o período que foi instituído 2011 (lançamento do programa) até o ano de 2016. As informações analisadas foram retiradas da base do Sistema de Informações Ambulatoriais do SUS SIASUS do MS, através do TabWin4, foram levantadas as variáveis: $1 . \mathrm{n}^{0}$ de equipes habilitadas; 2. população; 3. quantitativo de Emad I , Emad II e Emap implantadas; 4.

\footnotetext{
${ }^{4}$ Trata-se de aplicativos úteis na tabulação de dados e no cruzamento de informações de forma rápida, possibilitando aos gestores, estudantes e público em geral obter informações diversas no âmbito do SUS, sendo importante na gestão das políticas de saúde (DATASUS, 2010).
} 
custeio anual das SAD's; 5. leitos disponíveis do SUS ano; 6. $\mathrm{n}^{\circ}$ de internações domiciliares ano; 7. quantitativo real de internações no SUS ano; 8. Gasto média destas internações; 9. permanência média das internações; 10.gênero; e 11.faixa etária dos pacientes, sobre as bases de dados supracitadas, encontram-se disponíveis em: http://www.datasus.gov.br.

\section{RESULTADOS E DISCUSSÃO}

Desde seu lançamento em 2011 até 2016, tomando como base a Portaria GM/MS n 825 de 25 de abril de 2016 que "Redefine a Atenção Domiciliar no âmbito do Sistema Único de Saúde (SUS) e atualiza as equipes habilitadas", foram implantadas no Brasil 922, Emad I 515, Emad II 82 e 325 Emap em 340 municípios de 25 Unidades Federativas, representando uma cobertura populacional de cerca de $37,11 \%$ da população o que em números fica em torno de 77.075.667 (setenta e sete milhões, setenta e cinco mil, seiscentos e sessenta e sete) habitantes. Observa-se uma maior concentração do SAD na Região Sudeste com $41,76 \%$ do total, seguida em ordem decrescente pela Regiões Nordeste $32,97 \%$, Centro-Oeste $8,89 \%$, Sul $8,46 \%$ e Norte $7,92 \%$, conforme gráfico abaixo.

O Estado de Pernambuco na região nordeste representa 15,46\% do total de SAD implantados, e dos 181 municípios desses apenas 15 municípios possuem o SAD, conforme tabela a baixo.

Tabela 1. Quantitativo de SAD's habilitadas e implantadas no estado de Pernambuco até 25 de abril de 2016.

\begin{tabular}{|c|c|c|c|c|c|c|c|c|}
\hline \multicolumn{6}{|c|}{ QUATINTATIVO DE EQUIPES HABILITADAS } & \multicolumn{3}{|c|}{ CUSTEIO ANUAL } \\
\hline CODIGO DO IBGE & MUNICÍPIO & HABITANTES & EMAD 1 & EMAD 2 & EMAP & EMAD 1 (R\$) & EMAD 2 (R\$) & EMAP (R\$) \\
\hline 260005 & ABREU E LIMA & 98.201 & 1 & - & 1 & $600.000,00$ & - & $72.000,00$ \\
\hline 260050 & AGUAS BELAS & 42.291 & 1 & - & 1 & $600.000,00$ & - & $72.000,00$ \\
\hline 260290 & CABO DE SANTO AGOSTINHO & 95.979 & 1 & - & 1 & $600.000,00$ & - & $72.000,00$ \\
\hline 260410 & CARUARU & 277.982 & 3 & - & 1 & $1.800 .000,00$ & - & $72.000,00$ \\
\hline 260620 & GOIANA & 78.618 & 1 & - & 1 & $600.000,00$ & - & $72.000,00$ \\
\hline 260760 & ILHA DE ITAMARACA & 24.888 & - & 1 & 1 & - & $408.000,00$ & $72.000,00$ \\
\hline 260775 & ITAPISSUMA & 25.798 & - & 1 & 1 & - & $408.000,00$ & $72.000,00$ \\
\hline 260790 & JABOATAO DOS GUARARAPES & 335.371 & 5 & - & 2 & $3.000 .000,00$ & - & $144.000,00$ \\
\hline 260880 & LAJEDO & 39.240 & - & 1 & - & - & $408.000,00$ & - \\
\hline 261110 & PETROLINA & 217.093 & 3 & - & 1 & $1.800 .000,00$ & - & $72.000,00$ \\
\hline 261160 & RECIFE & 1.538 .000 & 9 & - & 3 & $5.400 .000,00$ & - & $216.000,00$ \\
\hline 261300 & SAO BENTO DO UNA & 47.230 & 1 & - & - & $600.000,00$ & - & - \\
\hline 261310 & SAO CAITANO & 36.859 & - & 1 & 1 & - & $408.000,00$ & $72.000,00$ \\
\hline 261330 & SAO JOAQUIM DO MONTE & 21.215 & - & 1 & 1 & - & $408.000,00$ & $72.000,00$ \\
\hline 261640 & VITORIA DE SANTO ANTAO & 11.541 & 1 & - & 1 & $600.000,00$ & - & $72.000,00$ \\
\hline
\end{tabular}


O Programa Melhor em Casa atendeu ao longo destes quatro anos (2013-2016) 23.231 pacientes, atingindo o seu auge em 2015, com 11.130 pessoas, segundo dados da Sala de Apoio à Gestão Estratégica - SAGE/Ministério da Saúde, alcançando sua maior representatividade.

Os 15 municípios de Pernambuco que compõem o SAD representam mais que a metade dos leitos disponíveis 52,91\%, ofertando um total de 110.014 leitos. A implantação do SAD no estado representou uma racionalização ao longo de sua existência de $23.231 \mathrm{mil}$ leitos no SUS em Pernambuco, confirmando a potencialidade do o atendimento domiciliar em desafogar a porta de entrada de urgência e emergência. Quando se observa que no espaço de seis anos (2011-2016) o serviço apresentou uma média de 29,59\% de internações acima do quantitativo de leitos ofertados.

A tabela a baixo faz uma correlação números de internações no SUS em relação ao numero de leitos e quanto percentualmente representa o incremento desse programa ao longo desse corte histórico.

Tabela 2. Analise dos números de internações SUS X SAD

\begin{tabular}{llllccrr}
\hline \multicolumn{1}{c}{ VARIÁVEIS } & $\mathbf{2 0 1 1}$ & $\mathbf{2 0 1 2}$ & $\mathbf{2 0 1 3}$ & $\mathbf{2 0 1 4}$ & $\mathbf{2 0 1 5}$ & $\mathbf{2 0 1 6}$ & TOTAL/MÉDIA \\
\hline \hline & & & & & & & \\
№ de Internações & 337.147 & 344.081 & 364.080 & 373.234 & 373.582 & 386.027 & $\mathbf{2 . 1 7 8 . 1 5 1}$ \\
№ de Leitos & 104.590 & 105.228 & 104.738 & 110.163 & 108.925 & 110.014 & $\mathbf{6 4 3 . 6 5 8}$ \\
\% da Relação Internações X Leitos & 31,02 & 30,58 & 28,77 & 29,52 & 29,16 & 28,50 & $\mathbf{2 9 , 5 9}$ \\
№ de Internações Domiciliares & $\ldots$ & $\ldots$ & $\mathbf{4 . 2 1 7}$ & $\mathbf{7 . 2 4 2}$ & $\mathbf{1 1 . 1 3 0}$ & $\mathbf{6 4 2}$ & $\mathbf{2 3 . 2 3 1}$ \\
\% da Relação Internações Domiciliares X Leitos & $\ldots$ & $\ldots$ & $\mathbf{4 , 0 3}$ & $\mathbf{6 , 5 7}$ & $\mathbf{1 0 , 2 2}$ & $\mathbf{0 , 5 8}$ & $\mathbf{5 , 3 5}$
\end{tabular}

Fonte: http://www.datasus.gov.br; http://sage.saude.gov.br/\# PORTARIA No 825, DE 25 DE ABRIL DE 2016| IBGE - 2017.

Em relação à tabela acima, pode-se ainda inferir que este incremento médio de 5,35\% de "leitos" oriundos do Programa Melhor em Casa, desafogou em 1,07\% a principal porta de entrada do SUS, frente ao cenário de superlotação das urgências e emergências no estado de Pernambuco, demonstrando que proposta de Atenção Domiciliar se torna algo viável e factível.

\section{CONCLUSÃO}

Os analisadores utilizados revelam aspectos importantes, que marcam a proposta do SAD. Pode-se afirmar que o serviço de atenção domiciliar tem de fato uma capacidade de descongestionar os hospitais e proporcionar a construção de nova lógica de atenção, racionalizar a utilização dos leitos hospitalares. SAD ganha força na agenda do SUS no estado, pela potencialidade na produção de novas formas de cuidar frente a um modelo de atenção ainda fragmentado e focado em procedimentos. 


\section{REFERÊNCIAS}

ALENCAR, VA. Contribuições da internação domiciliar em promover a desospitalização e prevenir a reospitalização no âmbito do SUS. 2013. 125 f., il. Dissertação (Mestrado em Enfermagem)—Universidade de Brasília, Brasília, 2013. Disponivel em: < http://repositorio.unb.br/handle/10482/15227 > Acesso em: 29. Abr. 2017

BRASIL. Ministério da Saúde. Secretaria de Atenção à Saúde. Departamento de Atenção Básica. Coordenação-Geral de Atenção Domiciliar. Manual de Monitoramento e Avaliação da Atenção Domiciliar/Melhor em Casa. Brasília: Ministério da Saúde, 2014.

BRASILa. Ministério da Saúde. Manual Instrutivo Melhor em Casa. 19p. Brasília, 2013.

BRASIL. Ministério da Saúde. Portaria No 825, de 25 de abril de 2016. Redefine a Atenção Domiciliar no âmbito do Sistema Único de Saúde (SUS) e atualiza as equipes habilitadas. Disponível em: < http://bvsms.saude.gov.br/bvs/saudelegis/gm/2016/prt0825_25_04_2016.html >. Acesso em : 12 de dez.2016.

BRASIL. Ministério da Saúde. Portaria. 1.533 de 16 de Julho de 2012. Redefine a Atenção Domiciliar no âmbito do Sistema Único de Saúde (SUS). 2012. Disponível em: < http://bvsms.saude.gov.br/bvs/saudelegis/gm/2012/prt1533_16_07_2012.html >. Acesso em : 12 de dez.2016.

BRASIL. Ministério da Saúde. Portaria MS/GM n ${ }^{0} 1.208$, de 23 de junho de 2013. Dispõe sobre a integração do Programa Melhor em Casa (Atenção Domiciliar no âmbito do SUS) com o Programa SOS Emergências, ambos inseridos na Rede de Atenção às Urgências. Diário Oficial [da] União, Brasília, DF, 23 jun. 2013(b). Disponível em: < http://bvsms.saude.gov.br/bvs/saudelegis/gm/2013/prt1208_18_06_2013.html $>$. Acesso em: 28 de dez. 2016.

GALVÃO, Claudia Raffa et al. Análise de indicadores de monitoramento de pacientes portadores de doenças crônicas: estratégia de redução de custos. Mundo saúde, v. 35, n. 4, p. 427-37, 2011. Disponível em: < https://www.saocamilosp.br/pdf/mundo_saude/88/09_AnalisedeIndicadoresdeMonitoramento.pdf > Acesso em: 27 abr. 2017.

OLIVEIRA NETO, Aristides Vitorino de. Análise do Programa Melhor em Casa: um olhar sobre a atenção domiciliar no âmbito do Sistema Único de Saúde (SUS). 2016. Disponível em: < http://periodicos.unifor.br/RBPS/article/view/4651 > Acesso em: 26 abr. 2017.

\section{AGRADECIMENTOS}

Agradecimento a secretaria de saúde do estado de Pernambuco, pela orientação quanto a manipulação do TBWEN, ao ministério da saúde pela disponibilização do banco de dados do programa melhor em casa. 